${ }^{1}$ Lourdes Jiménez Navascués

${ }^{2}$ Carlos A. Hijar Ordovas

${ }^{1}$ D.U.E., profesora de la E.U.E. de Soria.

${ }^{2}$ Oftalmólogo, Complejo Hospitalario SACYL, Soria.

Correspondencia:

Lourdes Jiménez Navascués

E.U. Enfermería

Ctra Logroño s/n. Soria-42004

Tfno: 6198801 28, 975233645

E-mail: lourji@enf.uva.es

\section{Los ancianos y las alteraciones visuales como factor de riesgo para su independencia}

\author{
Visual disorders as a risk factor for \\ elderly people's autonomy
}

\section{RESUMEN}

Introducción: El envejecimiento demográfico es una realidad en los países desarrollados, dando lugar a nuevas necesidades sociales y sanitarias entre el grupo de población de mayores de 65 años. Para dar respuesta alguna de estas necesidades se crean las instituciones gerontológicas de carácter sociosanitario. El presente estudio pretende conocer cómo las alteraciones visuales contribuyen en la autonomía de los ancianos residentes en un centro sociosanitario. Material y método: Estudio descriptivo transversal en el que se analizan en un grupo de residentes válidos: variables sociodemográficas, escalas de valoración funcional (Barthel, Lawton, etc.) y variables de aspectos oftalmológicos. Conclusiones: La alteración visual sí influye en la autonomía del individuo; observamos cómo a mayor edad, mayor es el grado de dependencia para las AVD. La patología ocular susceptible de ser tratada más frecuente es la catarata. No se emplean ayudas ópticas específicas para el trastorno de baja visión.

\section{PALABRAS CLAVE}

Dependencia, actividades de la vida diaria, alteración visual, ancianos, institución sociosanitaria, autonomía.

\section{SUMMARY}

Introduction: Demographic aging is a fact in developed countries, giving rise to new social needs in the group of population older than 65. In order to meet these needs, more gerontologic centers (both social and sanitary) are being created. Purpose: To determine whether visual disorders have an effect on the autonomy of the inmates in an elderly people's residence. Methods: A descriptive crosssectional study of the valid residents. Descriptive statistical analysis of socio-demographic variable, scales of functional valuation (Barthel, Lawton, etc.) and variables of ophthalmologic injuries. Conclusions: Visual disorders have an influence on the autonomy of people. We observed the older the age, the greater the degree of dependency for the activities of the daily life; the most frequent ocular pathology susceptible to be treated is cataract. Specific optical aids for the problems of impaired vision are not commonly used.

\section{KEY WORDS}

Dependency, activities of the daily life, visual change, autonomy, elder, geriatric center.

\section{INTRODUCCIÓN}

El envejecimiento demográfico es una realidad en gran parte del mundo, en el conjunto de los países desarrollados existen diferencias en la secuencia temporal y en la intensidad de este proceso. En la mayoría de los países de la Unión Europea la situación de envejecimiento era patente al inicio de los años setenta, en los países del sur europeo esta tendencia se conforma a finales de la década de los ochenta, este retraso frente a la mayoría de Europa se ha caracterizado por su extrema rapidez e intensidad (1).

Las estimaciones de población para el año 2020, son que el grupo de personas mayores de 65 años, constituirán más de un cuarto de la población de la Unión Europea (2). Según el último informe de prospectiva de $\mathrm{Na}$ ciones Unidas, se estima que España será el país “más vie- jo" del mundo en el 2050 (3). Ya hoy, nos encontramos en España con una esperanza de vida al nacer es de 78,03 años como media, para los varones, 74,44 años y para las mujeres, de 81,63. En Soria, donde realizamos el presente estudio, la esperanza de vida es ligeramente superior a la media nacional: 76,18 años para los varones, $83,76 \mathrm{pa}-$ ra las mujeres y el total, de 79,84 años (4).

Este desarrollo demográfico, unido a los cambios sociales sobre todo con la mutación del papel tradicional de la mujer, cuidadora habitual en el domicilio, que se ha incorporado activamente al mundo del trabajo y al conjunto de la vida social, ofrece como resultado una profunda transformación social. En este marco surgió el Plan Gerontológico Nacional (5), que pretende entre otros objetivos ser una guía para mejorar las condiciones de vida de 


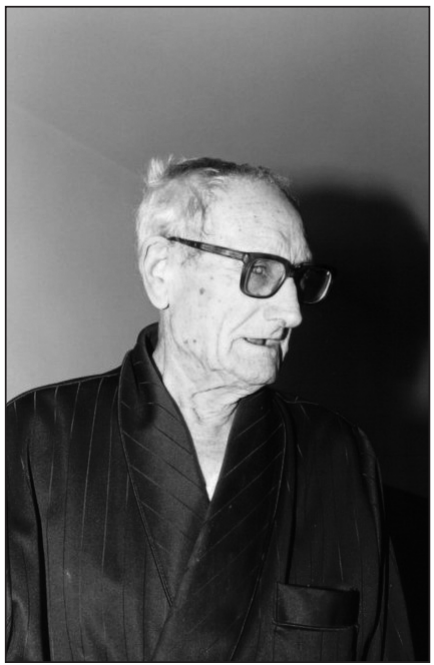

los mayores, poniendo a su disposición una red de recursos y reforzar su autonomía.

Se crean instituciones gerontológicas de carácter sociosanitario preocupadas por la promoción del bienestar del anciano, dan respuesta a las necesidades planteadas como consecuencia de los problemas sociales y/o sanitarios, que afectan a numerosas personas mayores. El declive físico asociado a la vejez conlleva necesidades de atención tanto psicosocial como sanitaria que ofrecen las residencias de ancianos. En el Estado español existen 2,53 plazas por cada 100 personas mayores, en nuestra comunidad autónoma la ratio asciende a $4,82(6)$.

Los cambios fisiológicos que implica el proceso de envejecimiento, generalmente generan un mayor grado de dependencia $(7,8)$ en las personas ancianas, desencadenando una serie de necesidades no cubiertas que han de ser satisfechas (9). La institucionalización de un anciano, es decir, su ingreso en una residencia, también se relaciona con el aumento en el grado de dependencia $(10,11)$. y la pérdida de autonomía se asocia a una peor calidad de vida $(12-15)$.

Un mejor conocimiento de los factores que influyen en la pérdida de autonomía de los ancianos institucionalizados, nos permitirá a los profesionales de Enfermería programar intervenciones de educación y promoción de la salud, prevención de la enfermedad y recuperación de la función perdida, disminuir y retrasar la dependencia de los ancianos, fomentar su autonomía y mejorar o mantener su calidad de vida. Competencias propias de la enfermera en residencias de ancianos, como prevé la Sociedad Española de Enfermería Geriátrica y Gerontológico (16).

El interés de nuestro estudio es conocer cómo las alteraciones visuales, influyen en la capacidad para realizar actividades de la vida diaria de los ancianos institucionalizados en un centro sociosanitario. Sabemos desde hace años que la pérdida de visión aumenta con el envejecimiento, siendo mayor en las personas ancianas que en los jóvenes (17-19).

Se ha relacionado la pérdida de visión con discapacidad para las actividades de la vida diaria; algunos estudios realizados en residencias de ancianos encuentran que los residentes con baja visión son dependientes en actividades como trasladarse de un lugar a otro, aseo corporal, etc. (20, 21). Otros estudios ponen de manifiesto que las áreas más afectadas por el déficit visual son la lectura, la movilidad fuera del hogar, las actividades de diversión y el salir de compras $(22,23)$. Las alteraciones de la conducta se han relacionado también con el déficit visual en residencias de ancianos (24), así como con las caídas de los ancianos (25); otros estudios muestran un descenso de la calidad de vida en las personas adultas con problemas visuales (26).

Las causas mas comunes de perdida de visión en los ancianos son la degeneración macular asociada a la edad (DMAE), la catarata, el glaucoma y la retinopatía diabética (27), siendo la catarata la primera causa de pérdida de visión en las personas mayores de 60 años y, en la mayoría de las ocasiones, susceptible de ser tratada con éxito. La recuperación de la función visual en estos pacientes ancianos tendrá unos efectos positivos sobre su calidad de vida (28).

\section{OBJETIVOS DE LA INVESTIGACIÓN}

\section{General}

Evaluar la influencia que ejercen las alteraciones visuales sobre la autonomía del anciano.

\section{Específicos}

Conocer el grado de dependencia en el momento de la valoración.

Identificar los residentes con trastornos visuales susceptibles de tratamiento.

Identificar el conocimiento que tienen de las ayudas ópticas disponibles en el mercado.

\section{MATERIAL Y MÉTODOS}

\section{Diseño}

Estudio descriptivo transversal.

Población de estudio

La muestra se compone de todos los ancianos ingresados en la Residencia mixta de Los Royales de Soria, perteneciente a la Gerencia de Servicios Sociales de la Junta de Castilla y León, considerados válidos, durante el período de tiempo comprendido entre el 1 de septiembre y el 30 de octubre del 2003. La población de estudio la componen 79 ancianos.

\section{Descripción del centro}

La Residencia para Mayores Los Royales, es de carácter público y depende de la Junta de Castilla y León, Consejería de Familia e Igualdad de Oportunidades, de la Gerencia de Servicios Sociales, según sus estatutos (29), es una unidad residencial considerada como un recurso de residencia habitual, preferentemente para las personas mayores, donde se les presta una atención continuada, integral y profesional durante las 24 horas del día. El acceso a las plazas en el centro residencial está regulado por Decreto 56/2001, de 8 de marzo (30), que define como plazas para personas mayores válidas aquellas en las que se presta atención a las personas mayores que mantienen unas condiciones personales, físicas y psíquicas, que les permiten realizar de forma autónoma las actividades básicas de la vida cotidiana. El número de plazas con las características citadas es de 90 . 
18 Procedimiento del estudio

Tras solicitar y obtener los permisos pertinentes mediante entrevista personal y solicitud escrita, del investigador con el director del centro y la responsable del área asistencial, informando de los objetivos del estudio, así como la metodología a seguir, se comenzó la captación de todos los residentes con cita previa del día y hora de la entrevista y exploración oftalmológica.

\section{Criterios de inclusión}

Se incluyeron todos las ancianos válidos ingresados en el momento de la recogida de datos.

\section{Criterios de exclusión}

Los ancianos que, aun cumpliendo los criterios de inclusión, no accedieron al estudio y todos los ancianos que ocupan plazas para personas mayores asistidas.

\section{DISEÑO ESTUDIO}

Variables sociodemográficas

Todos los datos necesarios para cumplimentar estas variables son obtenidas por el propio residente a través de una entrevista personal programada con anterioridad.

Las variables examinadas son:

- Sexo: hombre o mujer.

- Edad: en años.

- Número de años que lleva viviendo en la residencia.

- Antes del ingreso en la residencia jera independiente?

Variables que analizan la dependencia Grado de autonomía. Con esta variable pretendemos medir la capacidad funcional en cuanto a autonomía o dependencia que presenta el anciano/a referente a actividades cotidianas. Realizamos dos medidas, una recogiendo los datos aportados por el residente y otra es el personal de enfermería responsable de su cuidado quien nos proporciona los datos, esto nos permitirá estudiar si la autopercepción del anciano sobre su nivel funcional coincide con el de los profesionales que prestan los cuidados.

Índice de Barthel (31) e indice de Lawton (32)

Variables de aspectos oftalmológicos

1. Ayudas ópticas: ¿̨utiliza gafas? ¿¿tro tipo de ayuda óptica?

2. Agudeza visual monocular y binocular: con optotipos de Snellen, escala de 1 a 0.

3. Cataratas.

4. Degeneración macular asociada a la edad (DMAE): mediante fundoscopia.

5. Retinopatía diabética $(\mathrm{RD})$ : se diagnosticó mediante fundoscopia.

6. Presión intraocular (PIO): se midió mediante técnica de aplanación, con tonómetro manual Perkins.
ANÁLISIS

\section{DE LOS DATOS}

Con el programa SPSS.11 realizamos la estadística, un análisis descriptivo de las variables de estudio, frecuencias, tablas de contingencia.

Se analizó estadísticamente la asociación entre variables mediante el test de Chi cuadrado, análisis comparativo entre las diferentes escalas de valoración funcional y las distintas variables oftalmológicas considerando significación estadística para valores de $\mathrm{p}<0,05$.

Se realiza el test de Kappa para ver la concordancia entre las valoraciones de los individuos y los residentes en las escalas que valoran el grado de autonomía tanto para las actividades de la vida diaria como las instrumentales.

\section{RESULTADOS}

\section{Características sociodemográficas}

Del total de la muestra el 63,3\% son mujeres, con una edad media de 81,69 años, la edad media de los varones es de 80 años; en cuanto al número de años que llevan viviendo en la residencia, la media es de 3,37 años con una desviación típica de 1,57.

Con respecto a su situación funcional previa al ingreso en la institución, se valoraban como autónomos para realizar sus actividades el 94,7\% de las mujeres y el $84,2 \%$ de los varones (Tabla 1 ).

\section{Capacidad funcional}

En relación a la capacidad para realizar actividades básicas de la vida diaria, medida a través de la escala de Barthel, que también valora la autonomía de la persona anciana para realizar las actividades básicas e imprescindibles de la vida diaria, nos encontramos con los siguientes niveles de dependencia (Tabla 2).

Tabla 1. Distribución según dependencia antes del ingreso y sexo

\begin{tabular}{|l|l|l|l|}
\hline \multirow{2}{*}{ Independiente } & \multicolumn{2}{|c|}{ Sexo } & \multirow{2}{*}{ Total } \\
\cline { 2 - 3 } & Hombre & Mujer & \\
\hline Dependiente & 16 & 36 & 52 \\
& $84,2 \%$ & $94,7 \%$ & $91,2 \%$ \\
\hline Total & 3 & 2 & 5 \\
& $15,8 \%$ & $5,3 \%$ & $8,8 \%$ \\
& 19 & 38 & 57 \\
& $100,0 \%$ & $100,0 \%$ & $100,0 \%$ \\
\hline
\end{tabular}

Tabla 2. Escala de valoración de Barthel

\begin{tabular}{|l|l|l|l|l|l|c|}
\hline \multirow{2}{*}{} & \multicolumn{2}{|c|}{ Valoración del residente } & \multicolumn{3}{c|}{ Valoración de enfermería } \\
\cline { 2 - 3 } & \multicolumn{2}{|c|}{ Sexo } & \multirow{2}{*}{ Total } & \multicolumn{2}{c|}{ Sexo } & \multirow{2}{*}{ Total } \\
\cline { 2 - 6 } & Hombre & Mujer & & Hombre & Mujer & \\
\hline Dependencia severa & $3,4 \%$ & $2,0 \%$ & $2,5 \%$ & $10,3 \%$ & $8,0 \%$ & $8,9 \%$ \\
Dependencia moderada & $6,9 \%$ & $6,0 \%$ & $6,3 \%$ & $3,4 \%$ & $10,0 \%$ & $7,6 \%$ \\
Dependencia ligera & $51,7 \%$ & $54,0 \%$ & $53,2 \%$ & $58,6 \%$ & $36,0 \%$ & $44,3 \%$ \\
Indepencia & $37,9 \%$ & $38,0 \%$ & $38,0 \%$ & $27,6 \%$ & $46,0 \%$ & $39,2 \%$ \\
\hline
\end{tabular}


Vemos como la capacidad para realizar actividades instrumentales de la vida diaria, medida a través de la Escala de Law-

\section{Tabla 3. Escala de valoración Lawton}

\begin{tabular}{|c|c|c|c|}
\hline \multicolumn{4}{|l|}{ Lawton, residente } \\
\hline & \multicolumn{2}{|c|}{ Sexo } & Total \\
\hline & Hombre & Mujer & \\
\hline Dependencia total & $3,4 \%$ & $28,0 \%$ & $19,0 \%$ \\
\hline Dependencia severa & $13,8 \%$ & $2,0 \%$ & $6,3 \%$ \\
\hline Dependencia moderada & $24,1 \%$ & $22,0 \%$ & $22,8 \%$ \\
\hline Dependencia ligera & $13,8 \%$ & $26,0 \%$ & $21,5 \%$ \\
\hline Autónomo & $44,8 \%$ & $22,0 \%$ & $30,4 \%$ \\
\hline \multicolumn{4}{|l|}{ Lawton, enfermería } \\
\hline & \multicolumn{2}{|c|}{ Sexo } & Total \\
\hline & Hombre & Mujer & \\
\hline Dependencia severa & $37,9 \%$ & $34,0 \%$ & $35,4 \%$ \\
\hline Dependencia moderada & $20,7 \%$ & $18,0 \%$ & $19,0 \%$ \\
\hline Dependencia ligera & $24,1 \%$ & $32,0 \%$ & $29,1 \%$ \\
\hline Autónomo & $17,2 \%$ & $16,0 \%$ & $16,5 \%$ \\
\hline
\end{tabular}

Tabla 4. Escalas de valoración funcional con edad y número de años ingresados, valores de chi cuadrado

\begin{tabular}{|l|l|l|}
\hline & Edad & Años ingresados \\
\hline Katz personal & $\mathbf{0 , 0 3 1}$ & 0,753 \\
Katz cuidador & $\mathbf{0 , 0 0 1}$ & $\mathbf{0 , 0 4 7}$ \\
Barthel personal & $\mathbf{0 , 0 4 9}$ & 0,308 \\
Barthel cuidador & $\mathbf{0 , 0 1 0}$ & 0,528 \\
Lawton y Brody personal & $\mathbf{0 , 0 4 5}$ & 0,311 \\
Lawton y Brody cuidador & $\mathbf{0 , 0 4 9}$ & $\mathbf{0 , 0 2 6}$ \\
\hline
\end{tabular}

Tabla 5. Prevalencia de ceguera, baja visión y visión funcional relacionado con grupos de edad y sexo

\begin{tabular}{|l|l|l|l|l|}
\hline & Visión funcional & Baja visión & Ceguera & Total \\
\hline 60 a 69 años & $100,0 \%$ & $0,0 \%$ & $0,0 \%$ & $100,0 \%$ \\
70 a 79 años & $72,2 \%$ & $22,2 \%$ & $5,6 \%$ & $100,0 \%$ \\
80 a 89 años & $72,3 \%$ & $25,5 \%$ & $2,1 \%$ & $100,0 \%$ \\
90 ó más años & $42,9 \%$ & $57,1 \%$ & $0,0 \%$ & $100,0 \%$ \\
Hombre & $72,4 \%$ & $27,6 \%$ & $0,0 \%$ & $100,0 \%$ \\
Mujer & $71,4 \%$ & $24,5 \%$ & $4,1 \%$ & $100,0 \%$ \\
\hline Total & 56 & 20 & 2 & 78 \\
& $71,8 \%$ & $25,6 \%$ & $2,6 \%$ & $100,0 \%$ \\
\hline
\end{tabular}

\begin{tabular}{|l|l|l|l|l|l|}
\hline \multicolumn{7}{|c|}{ Tabla 6. Prevalencia de cataratas según edad } \\
\hline & \multicolumn{5}{|c|}{ Grupo de edad } \\
\cline { 2 - 5 } & 60 a 69 & 70 a 79 & 80 a 89 & 90 ó más & \multirow{2}{*}{ Total } \\
\hline No catarata clínica & $100,0 \%$ & $72,2 \%$ & $43,8 \%$ & $14,3 \%$ & $51,9 \%$ \\
Catarata & $0,0 \%$ & $27,8 \%$ & $54,2 \%$ & $85,7 \%$ & $46,8 \%$ \\
\hline Total & 6 & 18 & 48 & 7 & 79 \\
& $100,0 \%$ & $100,0 \%$ & $100,0 \%$ & $100,0 \%$ & $100,0 \%$ \\
\hline
\end{tabular}

ton y Brody, el 30\% de los residentes se consideran autónomos, mientras que el personal cuidador considera totalmente autónomo en estas actividades al 16,5\%. (Tabla 3)

$\mathrm{Al}$ realizar tablas de contingencia con las variables de edad y número de años ingresados en la residencia y las diferentes valoraciones funcionales, obtenemos los datos expresados en la Tabla 4: existe una relación proporcional entre el aumento del índice de dependencia y el incremento de la edad con significaciones de $\mathrm{p}<0,05$, en todas las escalas de valoración funcional.

\section{Alteraciones visuales}

De los residentes que se consideran con trastornos visuales, afirman el 68,6\% que tienen pequeños problemas para realizar actividades que ellos aprecian como habituales y el $26 \%$ dicen no saber si su problema tiene tratamiento. Como ayuda óptica el 70,9\% emplea las gafas.

Observamos que la baja visión aumenta progresivamente con la edad, de $0 \%$ en el grupo de edad de 60 a 69 años hasta el $57,1 \%$ en el grupo de mayor edad, no se observan diferencias significativas en relación con el sexo $(\mathrm{p}=$ $0,534)$. En la ceguera se observamos que los dos únicos residentes ciegos son mujeres con edades de 70 y 89 años.

De las patologías estudiadas la más frecuente en nuestra muestra es la catarata; la presentan el $46,8 \%$ de los residentes, seguida de la degeneración macular asociada a la edad (DMAE) con un 38\%, la retinopatía diabética con un 5\% y, por último, la hipertensión ocular con un 3,8\% (Tabla 5).

$\mathrm{Al}$ analizar los trastornos visuales con la edad y sexo nos encontramos con que la catarata aparece en el $44,8 \%$ de los hombres y en el $48 \%$ de las mujeres. Vemos un aumento progresivo proporcional a la edad, en el grupo de 60 a 69 años no se detecta a ningún residente, a los que tiene una edad entre 70 y 79 años presentan catarata el 27,8\%, aumentando al $54,2 \%$ en el grupo de 80 a 89 años y en el grupo de mayor edad es del $85,7 \%$, siendo una diferencia estadísticamente significativa $p=0,02$. (Tabla 6).

En el análisis de la degeneración macular asociada a la edad, la retinopatía diabética y a la hipertensión ocular no encontramos diferencias estadísticamente significativas en cuanto al sexo y grupos de edad.

Al estudiar los trastornos visuales con las escalas de valoración funcional encontramos los siguientes datos —valores estadísticamente significativos de $\mathrm{p}<0,05-$ (Tabla 7).

Se obtiene el valor de Kappa para ver si hay con- 
20 cordancia entre las valoraciones de los individuos y los cuidadores, y vemos que es significativo (los valores superiores a 1,96 son significativos), así como la chi cuadrado, nos indica dependencia entre ambas clasificaciones ( $\mathrm{Ta}-$ blas 8 y 9 ).

Tabla 7. Valoración oftalmológica y valoración funcional, valores de chi cuadrado

\begin{tabular}{|l|l|l|l|l|l|} 
& DMAE & RD & Visión & Catarata & $\begin{array}{l}\text { Hipertensión } \\
\text { ocular }\end{array}$ \\
\hline Barthel personal & 0,859 & 0,175 & 0,055 & 0,052 & 0,943 \\
Barthel cuidador & 0,585 & 0,010 & 0,002 & 0,089 & 0,841 \\
Lawton y Brody personal & 0,826 & 0,208 & 0,032 & 0,003 & 0,887 \\
Lawton y Brody cuidador & 0,359 & 0,376 & 0,191 & 0,107 & 0,367 \\
\hline
\end{tabular}

Tabla 8. Análisis asociación del Lawton entre personal y cuidadores

\begin{tabular}{|c|c|c|c|c|c|c|}
\hline & & \multicolumn{2}{|l|}{ Valor } & \multicolumn{3}{|c|}{ Sig. asintótica (bilateral) } \\
\hline & Chi cuadrado de Pearson & \multirow{2}{*}{\multicolumn{2}{|c|}{$\begin{array}{l}97,830 \\
0,642 \\
\end{array}$}} & \multirow{2}{*}{\multicolumn{2}{|c|}{$\begin{array}{l}0,000 \\
0,000\end{array}$}} & \\
\hline & V de Cramer & & & & & \\
\hline & & \multicolumn{5}{|c|}{ Lawton cuidadores } \\
\hline & & $\begin{array}{l}\text { Dependencia } \\
\text { severa }\end{array}$ & $\begin{array}{l}\text { Dependencia } \\
\text { moderada }\end{array}$ & $\begin{array}{l}\text { Dependencia } \\
\text { ligera }\end{array}$ & Autónomo & Total \\
\hline \multirow[t]{6}{*}{ Lawton personal } & Dependencia total & $\begin{array}{l}15 \\
4,2\end{array}$ & $\begin{array}{l}0 \\
-1,7\end{array}$ & $\begin{array}{l}0 \\
-2,1\end{array}$ & $\begin{array}{l}0 \\
-1,6\end{array}$ & 15 \\
\hline & Dependencia severa & $\begin{array}{l}5 \\
2,4\end{array}$ & $\begin{array}{l}0 \\
-1,0\end{array}$ & $\begin{array}{l}0 \\
-1,2\end{array}$ & $\begin{array}{l}0 \\
-, 9\end{array}$ & 5 \\
\hline & Dependencia moderada & $\begin{array}{l}6 \\
-0,2\end{array}$ & $\begin{array}{l}8 \\
2,5\end{array}$ & $\begin{array}{l}4 \\
-0,5\end{array}$ & $\begin{array}{l}0 \\
-1,7\end{array}$ & 18 \\
\hline & Dependencia ligera & $\begin{array}{l}1 \\
-2,0\end{array}$ & $\begin{array}{l}2 \\
-0,7\end{array}$ & $\begin{array}{l}14 \\
4,1\end{array}$ & $\begin{array}{l}0 \\
-1,7\end{array}$ & 17 \\
\hline & Autónomo & $\begin{array}{l}1 \\
-2,6 \\
\end{array}$ & $\begin{array}{l}5 \\
0,2 \\
\end{array}$ & $\begin{array}{l}5 \\
-0,8 \\
\end{array}$ & $\begin{array}{l}13 \\
4,6 \\
\end{array}$ & 24 \\
\hline & Total & 28 & 15 & 23 & 13 & 79 \\
\hline
\end{tabular}

\begin{tabular}{|c|c|c|c|c|c|c|c|c|}
\hline & & Valor & \multicolumn{2}{|c|}{ Error típ. asint. } & \multicolumn{2}{|c|}{$\mathrm{T}$ aproximada } & \multicolumn{2}{|c|}{ Sig. aproximada } \\
\hline & $\begin{array}{l}\text { Medida de acuerdo Kappa } \\
\text { N de casos válidos }\end{array}$ & $\begin{array}{l}0,563 \\
79\end{array}$ & \multicolumn{2}{|c|}{0,078} & 6,819 & & \multicolumn{2}{|c|}{0,000} \\
\hline & & \multicolumn{3}{|l|}{ Valor } & \multicolumn{4}{|c|}{ Sig. asintótica (bilateral) } \\
\hline & $\begin{array}{l}\text { Chi-cuadrado de Pearson } \\
\text { V de Cramer }\end{array}$ & \multicolumn{3}{|l|}{$\begin{array}{l}73,372 \\
0,556\end{array}$} & \multicolumn{4}{|l|}{$\begin{array}{l}0,000 \\
0,000\end{array}$} \\
\hline & & \multicolumn{7}{|c|}{ Barthel cuidadores } \\
\hline & & $\begin{array}{l}\text { Dependencia } \\
\text { severa }\end{array}$ & $\begin{array}{l}\text { Dependencia } \\
\text { moderada }\end{array}$ & \multicolumn{2}{|c|}{$\begin{array}{l}\text { Dependencia } \\
\text { ligera }\end{array}$} & \multicolumn{2}{|c|}{ Independencia } & Total \\
\hline \multirow[t]{5}{*}{ Bartel personal } & Dependencia severa & $\begin{array}{l}2 \\
4,3\end{array}$ & $\begin{array}{l}0 \\
-0,4 \\
\end{array}$ & \multicolumn{2}{|l|}{$\begin{array}{l}0 \\
-0,9\end{array}$} & \multicolumn{2}{|l|}{$\begin{array}{l}0 \\
-0,9\end{array}$} & 2 \\
\hline & Dependencia moderada & $\begin{array}{l}2 \\
2,3\end{array}$ & $\begin{array}{l}2 \\
2,6\end{array}$ & \multicolumn{2}{|l|}{,- 8} & & 5 \\
\hline & Dependencia ligera & $\begin{array}{l}3 \\
-0,4 \\
\end{array}$ & $\begin{array}{l}4 \\
0,5\end{array}$ & \multicolumn{2}{|l|}{$\begin{array}{l}29 \\
2.4\end{array}$} & \multicolumn{2}{|l|}{$\begin{array}{l}6 \\
-2,6\end{array}$} & 42 \\
\hline & Independencia & $\begin{array}{l}0 \\
-1,6\end{array}$ & $\begin{array}{l}0 \\
-1,5\end{array}$ & \multicolumn{2}{|l|}{$\begin{array}{l}5 \\
-2,3 \\
\end{array}$} & \multicolumn{2}{|l|}{$\begin{array}{l}25 \\
3,9\end{array}$} & 30 \\
\hline & Total & 7 & 6 & \multicolumn{2}{|l|}{35} & \multicolumn{2}{|l|}{31} & 79 \\
\hline
\end{tabular}


mujeres y el porcentaje se eleva al $70 \%$ si nos centramos en las personas de más de 85 años para el mismo período (34). Así pues nos encontramos con que las mujeres ocupan un papel central en cuanto a principales consumidoras de servicios sociosanitarios.

Esto nos hace reflexionar sobre si el proceso de envejecimiento es igual para hombres que para mujeres, ya que son pocos los estudios que profundizan en el tema del envejecimiento relacionado con el género y muchos los factores sociales que han podido contribuir a estas diferencias, como por ejemplo, la vivencia de la Guerra Civil española: en el reportaje "La mujeres que perdieron la guerra”, Dulce Chacón (35) pone de manifiesto en un medio de comunicación cómo muchas mujeres sufrieron como nadie y algunas han tenido que callar, no han realizado un trabajo asalariado, o han renunciado a él en el momento de contraer matrimonio y, por tanto, no tienen derecho a una pensión por jubilación, etc.

García Laborda (36) hace referencia a este mismo hecho y considera que una parte importante de las consecuencias del envejecimiento de la población es diferente para hombres y mujeres. Por tanto, consideramos que sería importante analizar estas diferencias y los factores que influyen en ello; desde el punto de vista de los cuidados, conocer los factores que hacen que cada anciano/a viva su vejez de manera diferente nos ayudará a planificar cuidados que mejoren la calidad de vida de los ancianos, entendiendo ésta como la movilización de los recursos que cada individuo tiene para satisfacer sus necesidades, añadir vida a los años significa, ayudar a las personas a disfrutar de $s u$ vida y obtener satisfacción de ella (37). En el estado de salud de los mayores intervienen múltiples factores, entre ellos las situaciones de riesgo a los que la persona ha estado expuesta durante su vida, sus hábitos y sus comportamientos (38), que han podido ser diferentes entre hombres y mujeres.

Cabe destacar que los varones tienen una media en relación al número de años que llevan ingresados, ligeramente superior a las mujeres a pesar de tener una edad media inferior, por lo que deducimos que el varón solicita el ingreso en la residencia con menor edad; además, los varones se consideraban más dependientes antes del ingreso, estos datos coinciden con el trabajo de Rojas y cols. (12); puede deberse a que desde siempre la mujer ha ejercido el papel de cuidadora, así como de realizadora de tareas del hogar, por tanto, ellas permanecen mayor tiempo en su hogar antes de ingresar en una institución, ya que estos datos se refieren a residentes considerados válidos.

Cuando evaluamos la capacidad funcional descrita por el propio anciano y la realizada por las enfermeras, aunque estadísticamente no hay diferencias significativas y en el análisis de Kappa observamos una significación entre ambas valoraciones, nos parece interesante destacar cómo la autopercepción de los residentes, en cuanto a las valoraciones de autonomía más extremas, tanto de independencia total como el mayor grado de dependencia, difieren con la percepción de sus cuidadores.
Se observa una relación directamente proporcional en- 21 tre el grado de dependencia de las actividades de la vida diaria y el incremento de la edad, coincidiendo con varios autores $(10,13,39,40)$. No existe, sin embargo, diferencias estadísticamente significativas en el número de años que lleva ingresado el anciano en la residencia y los valores de Katz tanto personal como de los cuidadores ni con Lawton y Barthel valorados por el personal de enfermería, estos datos contrastan con los aportados en su estudio por Rojas Ocaña y cols. (12), en el que constatan cómo el ingreso de un anciano válido en una institución conlleva un aumento en el nivel de dependencia.

Encontramos en nuestros resultados una prevalencia de la baja visión (25,6\% de los residentes) similar a la que encuentran otros autores en estudios similares al nuestro como el de Baltimore (41), que encuentra el $23 \%$. Observando, al igual que ellos, la existencia de una fuerte asociación entre la baja visión y la edad de los residentes. Sin embargo, no se utilizan ayudas ópticas específicas para corregir este defecto visual.

En cuanto a la ceguera, encontramos en nuestro estudio una prevalencia del 2,6\%, inferior al estudio de $\mathrm{Bal}-$ timore $^{50}(7,4 \%)$ y al Beaver Dam Eye Study (22) (8,3\%); posiblemente las personas ciegas mayoritariamente sean admitidas como residentes no válidos que, por tanto, han sido excluidos de nuestro estudio. Observamos un pico más alto en la prevalencia de la ceguera entre los sujetos de 70 a 79 años, parecido al encontrado por dichos autores, que pudiera reflejar el grado de importancia de la ceguera como necesidad de utilizar los recursos socio-sanitarios, en edades inferiores. $\mathrm{Y}$ al igual que en otros estudios (42), las mujeres tienen mayor ratio de ceguera que los hombres.

La patología ocular más frecuente encontrada es la catarata al igual que en los realizados en residencias y en la población general, siendo nuestra tasa más alta que la del estudio de Baltimore ( $46,8 \%$ versus $30 \%$ ), es una patología con la que no hemos encontrado relación estadísticamente significativa con el nivel de autonomía, posiblemente no exista afección binocular; por tanto, el individuo se maneja bien con la visión del ojo sano. Las otras patologías presentan también prevalencias similares a dichos estudios, excepto en la más alta prevalencia de la degeneración macular asociada a la edad, hallada por nosotros (38\% frente a $11,8 \%$ en el estudio de Baltimore).

$\mathrm{La}$ alta prevalencia de enfermedades oculares que pueden ser tratadas satisfactoriamente (como las cataratas) y que causan pérdida de visión, con las implicaciones en la calidad de vida de las personas que las padecen, sugiere un estudio de los adecuados programas de salud visual en las residencias de ancianos.

Destacar que la DMAE y la hipertensión intraocular no tienen una relación significativamente estadística con las escalas de valoración funcional; esto podría ser debido a que son patologías que clínicamente no dan manifestaciones en fases iniciales, y cuando realmente interfieran 
22 con las actividades del anciano es en estadios finales, difícilmente tratables satisfactoriamente. Esto justificaría la elaboración de un programa de detección precoz; en su publicación Jaén Díaz y cols. (43) también concluyen que se debería valorar la detección precoz de la hipertensión intraocular.

En nuestro estudio la baja visión es el trastorno visual que más interfiere en la autonomía para las actividades básicas de la vida diaria; otros estudios también han encontrado resultados similares $(24,25)$. Estos datos nos sugieren la posibilidad de profundizar sobre el conocimiento y utilización, que se tiene sobre los dispositivos de ayuda óptica existentes en el mercado, como podrían ser las telelupas. Los residentes encuestados sólo emplean las gafas como medio de ayuda óptica, destaca que el $26 \%$ dice no conocer si su trastorno tiene algún tipo de tratamiento.

Sobre la calidad de vida del anciano, a pesar de lo subjetivo del término, hay varios estudios que la relacionan, entre otros motivos, con la conservación de la capacidad funcional para mantener la autonomía personal y poder llevar a cabo las actividades de la vida diaria (14-16, 44, $45)$, o con los problemas visuales (30). En nuestra sociedad la independencia es uno de los signos de la valoración de la persona, la mayoría de las personas aspiramos a una vida libre de dependencia y son muchos los que manifiestan temor a esta situación. Con los datos recogidos podemos deducir que las personas a partir de los 80 años perdemos niveles de calidad de vida, puesto que la dependencia para las AVD se hace más manifiesta. Desde enfermería, se hace imprescindible trabajar por aumentar la esperanza de vida libre de dependencia, como dice Soldevilla (46), este reto se cierne sobre nosotros.

Prevenir la pérdida de autonomía y enseñar cómo vivir con dependencia son aspectos muy importantes a desarrollar por los profesionales de enfermería. La toma de decisiones del equipo de salud, sobre la actuación y desarrollo de cuidados en las personas mayores debe realizarse siempre con unas valoraciones previas, donde se incluyan los factores que influyen en la capacidad funcional, la investigación permanente sobre estos factores contribuirán a una mejora en la calidad asistencial que ofrecemos a los ancianos y contribuiremos sin duda a mejorar su calidad de vida.

\section{CONCLUSIONES}

La alteración visual que más afecta a la autonomía del individuo para las actividades de la vida diaria tanto las denominadas básicas, medidas por las escalas de Barthel y Katz, como las denominadas instrumentales, medidas por el test de Lawton y Brody, es la baja visión.

El grado de autonomía del individuo es directamente proporcional a la edad: a mayor edad, mayor dependencia para las actividades de la vida diaria básica.

La patología ocular más frecuente es la catarata, siendo susceptible de tratar con éxito de recuperación visual en la mayoría de los casos.

Se puede frenar el avance de la degeneración macular asociada a la edad, la retinopatía diabética y la hipertensión intraocular, si son detectadas en los primeros estadios; por ello, se deben diseñar programas de detección precoz.

Es preciso potenciar la utilización de ayudas ópticas específicas para el trastorno de baja visión.

\section{AGRADECIMIENTOS}

En primer lugar, a todos los ancianos de la Residencia de Los Royales, ya que sin ellos no tendría sentido nuestro trabajo. También un agradecimiento muy especial a los responsables de este centro, Da Carmen Alvarez y D. Antonio Valdenebro por su valiosa, desinteresada y constante colaboración.

\section{BIBLIOGRAFÍA}

1. Uranga Iturriotz MJ, Calvo Aguirre JJ, Torrijos Torrijos M. Enfermería Geriátrica en el medio sociosanitario. SEEGG: Donostia-San Sebastián; 2001.

2. Comité consultivo para la formación de enfermeros. Informe y recomendaciones sobre directrices para la formación en atención de Enfermería a personas mayores. Bruselas: Comisión Europea; 1995.

3. Marina JA. Una filosofía de la ancianidad. Rev Rol Enf 2004; 27 (4): 5-20.

4. Instituto Nacional de Estadística. Esperanza de vida, datos de 1995. URL: www.ine.es/inebase/cgi/axi

5. Plan Gerontológico. Instituto Nacional de Servicios Sociales. Ministerio de Asuntos Sociales Instituto Nacional de Servicios Sociales; Madrid 1993.

6. Las personas mayores en España. Informe 2000. IMSERSO 2000. www.seg-social.es/imserso/mayores/docs/io_maydesto.htm

7. Isla Pera P. El envejecimiento de la población. Rev Rol Enf 200326 (3): 15-20.

8. Fontana Fortuni C, Estany Almirall J, Pujol Salud J, Segarra Solanes I, Jordán Gómez Y. Concordancia entre índices de dependencia en las actividades de la vida diaria. Experiencia de aplicación en población geriátrica de ámbito rural. Enferm Clínica 2002; 12 (2): 47-53.

9. Quintanilla Martínez M. Anciano Frágil. Rev Rol Enf 2004; 27 (4): 275-280.
10. Rojas Ocaña, Toronjo Gómez A, Rodríguez Rodríguez JB, Abreu Sánchez A, Rodríguez Pérez M, López Santos MV. Ancianos válidos institucionalizados. Gerokomos 2004; 15 (1): 6-17.

11. Carmenaty Díaz, Idalmis y Soler Orozco L. Evaluación funcional del anciano. Rev Cubana Enfermer 2002; 18 (3): 184-8.

12. Martín Carrasco M, Oslé C, García P, Mosquera JL, Salvadó Gallén I, Las heras T, Aizcorbe C. Calidad de vida y estado de salud en las residencias geriátricas. Cuad Gerontológico 2001; 1 (1): 5-30.

13. Álvarez MA, Quadros Seiffert R, Takase Gonçalves. Calidad de vida en la vejez. Gerokomos 1998; 9 (3): 107-14.

14. Canga Armador, AD. Ancianos institucionalizados ¿̨cómo se percibe la vejez? Rev Rol Enf 2000; 23 (12): 841-7.

15. Rodríguez Gómez C, García Jardón S, Barbosa Alvarez MA, Tresandi PMA. Necesidades básicas en ancianos de residencias y asilos de Vigo y Tuy. Gerokomos 1993, 4 (9): 122-7.

16. Competencias de la enfermera en las residencias de ancianos. Documento técnico no 1 . Sociedad Española de Enfermería Geriátrica y Gerontológica. Noviembre 2002.

17. Tielsh JM, Sommer A, Witt K, Katz J, Royall RM. Blindness and visual impairment in an American urban population: the Baltimore Eye Survey. Arch Ophthalmol 1990; 108: 286-90.

18. Klein R, Klein BEK, Linton KLP, De Mets DL. The Beaver Dam Eye Study: visual acuity. Ophthalmology 1991; 98: 1310-5. 
19. Leiboiwitz HM, Krueger DE, Maunder LR, et al. The Framingham Eye Study monograph: an ophthalmological and epidemiological study of cataract, glaucoma, diabetic retinopathy, macular degeneration and visual acuity in a general population of 2631 adults, 1973-1975. Surv Ophthalmol 1980; 24 (Suppl): 335-610.

20. Marx MS, Werner P, Cohen-Mansfield J, Feldman R. The relationship between low vision and performance of activities of daily living in nursing home residents. J Am Geriatr Soc 1992; 40 (10): 1018-20.

21. Horowitz A. Vision impairment and functional disability among nursing home residents. Gerontologist 1994; 34 (3): 316-23.

22. Lamoureux EL, Hassell JB, Keeffe JE. The determinants of participation in activities of daily living in people with impaired vision. Am J Ophtalmol 2004; 137 (2): 265-70.

23. Rodríguez Ávila N. Dependencia al envejecer. Rev Rol Enf 2004; 27 (4): 262-272.

24. Horowitz A. The relationship between vision impairment and the assessment of disruptive behaviors among nursing home residents. Gerontologist 1997; 37 (5): 620-8.

25. Ivers RQ, Cumming RG, Mitchell P, Attebo K. Visual impairment and falls in older adults: the Blue Mountains Eye Study. J Am Geriatr Soc 1998; 46 (1): 58-64.

26. Broman A, Muñoz B, Rodríguez J, Sánchez R, Quigley H, Klein R et al. The impact of visual impairment and eye disease on vision-related quality of live in a Mexican-American population: proyecto VER. Invest Ophthalmol Vis Sci 2002; 43 (11): 3393-98.

27. Harvey PT. Common eye diseases of elderly people: identifying and treating causes of vision loss. Gerontology 2003; 49 (1): 1-11

28. Leske MC, Chilack LT, Wu SY. The lens opacities case-control study: risk factors for cataract. Arch Ophthalmol 1991; 109: 244-51.

29. Estatuto básico de Centros de Personas Mayores de Castilla y León. 2002 Junta de Castilla y León, Consejería de Sanidad y Bienestar Social Gerencia de Servicios Sociales.

30. Decreto 56/2001, de 8 de marzo, por el que se aprueba el reglamento regulador del régimen de acceso a las plazas en los centros residenciales para personas mayores, dependientes de la administración de la comunidad de Castilla y León y a las plazas concertadas en otros establecimientos. BOCYL no 57, de 20 de marzo de 2001.

31. Escalas de valoración geriátrica. Novartis 2003. Reproducción autorizada por: Mahoney FL, Barthel DW: Funcional evaluation: The Barthel Index. State Journal 1965; 4 (2): 61-5.
32. Escalas de valoración geriátrica. Novartis 2003. Reproducción autorizada por: The Gerontological Society of America. Gerontologist; assessment of older people: self-maintaining and instrumental activities of daily living. Lawton MP, Brody EM. 1969, 9 (3): 179-86.

33. Martín Carrasco M, Oslé C, García P, Mosquera JL, Salvadó Gallén I, Laceras T, Aizcorbe C. Calidad de vida y estado de salud en las residencias geriátricas de Pamplona. Cuad Gerontológico 2001; 1 (1): 5-30.

34. Santos del Campo I. Envejecimiento demográfico. Diferencias por género. Reis 1996; 73: 177-90.

35. Chacón D. Las mujeres que perdieron la guerra. El País Semanal 2002; 1353: 46-53.

36. García Laborda A. Envejecimiento y género: aspectos sociales. Rev Metas 2000; 26: 17-22.

37. Bonafont A. El cuidar y los ancianos. Gerokomos 1997; 8 (20): $49-59$.

38. García Llopis, AM. Programa de promoción de la actividad física en el anciano. Metas Enfermer 2002; V (50): 56-60.

39. Álvarez Solar M, de Alaiz Rojo AT, Brun Gurpegui E, Cabaneros Vicente JJ, Calzón Frechoso M, Cosío Rodríguez I et al. Capacidad funcional de pacientes mayores de 65 años, según el índice de Katz, fiabilidad del método. Atención primaria 1992; 10: 812-5.

40. Palacios Ceña D. Elementos que modifican la valoración del anciano frágil. Gerokomos 2003; 14 (3): 121-6.

41. James M, Tielsch PhD, Jonathan C, Javitt MD, Anne Coleman MD, Joanne Katz ScD, Alfred Sommer MD. The prevalence of Blindness and visual impairmente among nursing home residents in Baltimore. New Eng J Med 1995; 332 (18): 1205-9.

42. Salive ME, Guralnik J, Christen W, Glynn RJ, Colsher P, Osfeld AM. Funtional Blindness and visual impairment in older adults from three communities. Ophthalmology 1992; 99: 1840-7.

43. Jaén Díaz JL, Sanz Alcolea I, López de Castro F, Pérez Martínez T, Ortega Campos P, Corral Morales R. Glaucoma e hipertensión ocular en atención primaria. Atención Primaria 2001; 28 (1): 23-30.

44. Casado JM, González N, Moraleda S, Orueta R, Carmona J, GómezCalcerrada RM. Calidad de vida relacionada con la salud en pacientes ancianos en atención primaria. Atención primaria 2001; 28 (3): 167-73.

45. Pont Ribas A, Granollers Mercader S. Calidad de vida en los ancianos, un estudio descriptivo. Rev ROL Enferm 1992; 15 (165): 21-6.

46. Soldevilla Agreda JJ. Esperanza de vida libre de dependencia. Geroko$\operatorname{mos} 2002 ; 13$ (4): 176. 